\title{
Comparison of Different Types of Cutting Devices in Surgery (Review)
}

\author{
Victor Aleksandrovich Stupin ${ }^{1,2 *}$, Natalia Evgenievna Manturova ${ }^{1,2}$, Aleksei Alekseevich Donskikh ${ }^{3}$, Ekaterina \\ Vladimirovna Silina ${ }^{3}$
}

${ }^{I}$ Pirogov Russian National Research Medical University (RNRMU), Ostrovityanova St. 1, Moscow 117997, Russia

${ }^{2}$ Institute of Plastic Surgery and Cosmetology; Olkhovskaya St., 27, Moscow, 105066, Russia

${ }^{3}$ I.M. Sechenov First Moscow State Medical University (Sechenov University); Trubetskaya St., 8, Moscow, 119991, Russia

*Corresponding author E-mail: stupin.v.a@mail.ru

\begin{abstract}
The present work presents a description of various cutting devices with an emphasis on skin surgery for the optimal choice of instruments in plastic surgery. Physical principles of work with a description of the biological role of an ultrasonic scalpel, various laser cutting devices, electrosurgical instruments, including radiofrequency electrosurgery, are described. Studies of recent years, including systematic reviews and meta-analyzes, are devoted to comparative analysis of various cutting and coagulating devices among themselves and in comparison with the mechanical metal scalpel.
\end{abstract}

Keywords: Electrosurgery; Knife; Laser surgery; Plastic surgery; Radio wave surgery; Radiofrequency electrosurgery; Scalpel; Skin; Wound healing; Ultrasound scalpel.

\section{Introduction}

The last two decades have been marked by a significant increase in the number of services provided in the field of cosmetology and plastic surgery. For example, in the USA in 2014, 15.6 million various cosmetic procedures (including minimally invasive (e.g., involving Botox) and surgical ones) were performed. Their total cost was $\$ 12.9$ billion, which was 3\% more than in $2013[1,2]$. The interest of the population of economically developed countries in cosmetic plastic surgery is growing. The results of the online survey "ELLE/MSBNC.com Sex and Body Image Survey", including the data from 52,677 people aged from 18 to 65 years [3], showed that $48 \%$ of women were interested in cosmetic surgery or sometimes used it. Among men, only 23\% showed interest in cosmetology surgery. Age differences were not identified, while older women showed the greatest confidence in their decision to make a plastic surgery. These trends cause the fact that plastic surgery sets ever higher demands for the formation of the cosmetic cutaneous scar. To a certain extent this is what determines the ongoing search for a high-tech surgical instrument for small and large plastic surgeries.

The process of restoration of the operating wound is multifactorial. It is known that fibroblasts and keratinocytes predominate in the skin of mammals and humans; therefore, the result of the wound process will depend on their availability and condition [4]. The wound process is divided into separate phases: inflammation phase (the period of vascular changes and purification of the wound from necrotic/dead tissues, vasoconstriction, followed by vasodilation with the release of blood elements, WBC infiltration of the wound, phagocytosis); regeneration and proliferation phases (formation of a fibrin matrix for the migration of fibroblasts and epithelial cells to the healing wound, neoangiogenesis); and scar epithelialization and remodeling (accumulation of collagen in the wound, migration of keratinocytes from the wound edges, tissue remodeling with an increase in the strength of the scar tissue, scar restructuring with the formation of elastic fibers and the development of a new fibrotic network).

If the skin is damaged, all its 3 layers (epidermis, dermis, fatty tissue) are involved in a complex of physiological local and general body reactions aimed at restoration of the functional and anatomical integrity of the damaged tissues. As a rule, the scar is not formed only in case of superficial skin wounds' healing without disturbance of the basal layer of epidermis. That is, incisions of the papillary layer of the dermis, where grooves are formed and papillary skin pattern is formed, as well as the mesh layer of the dermis, in which elastic and collagen fibers form a network of frame matrix, heal without a trace. In other cases, the wound heals with the formation of connective tissue scar. However, the severity of this scar differs greatly depending on various factors (the size and nature of the lesion, morphological structure of the damaged tissues, infection of the wound, common condition of the patient and concomitant diseases, medicines used, the technique of surgical incision and wound suturing, methods of wound forming, agents, ointments, bandages used for wound healing, and much more).

Choice of proper cutting devices is one of the important factors affecting the healing of a postoperative wound.

The aim of this work was to search for, describe and evaluate scientific papers on the impact of different types of cutting devices used in surgery for skin incisions.

\section{Methods}

The following literature sources were investigated: PubMed/MEDLINE, Scopus, Cochrane Library. We searched through all publications in English-language journals over the past 
10 years, using only the following keywords and their combinations: scalpel, laser, ultrasound, electrosurgery, electrosurg*, radio*, device, surgery, wound, skin. The research works made an accent on the medical clinical and experimental studies devoted to the healing of wounds after skin incisions with different surgical instruments with the aim of the subsequent use of the work results in aesthetic medicine.

\section{Results}

The results of the work are presented in the form of sequential description of works devoted to ultrasound, laser scalpels, electrosurgery and radio wave knives.

The appearance of ultrasonic and laser scalpels in the 1950s helped to solve a number of problems. Especially great advances in the accurate coagulation of bleeding were reached in endoscopic surgery. However, despite the large number of such devices, there is still no consensus among surgeons about the use of these cutting tools

Ultrasonic scalpel cuts tissue with the frequency of $10-100 \mathrm{kHz}$ and an amplitude of 5-50 $\mu \mathrm{m}$. Its mechanism of action is based on two principles: mechanical (destruction of intercellular links due to vibration) and cavitational (as a result of exposure to highfrequency oscillations on tissues, a negative pressure is created on the tissues by a short time, which leads to the boiling of intracellular and intercellular fluid at the temperature of $38^{\circ} \mathrm{C}$. The resulting vapor destroys cell membranes and, spreading through the intercellular spaces, divides the tissues). An ultrasonic knife is very convenient to carry out separation of tissues and pathologically altered structures from normal ones, which is expedient for scars' excision.

In a randomized trial, Schneider $\mathrm{D}$ et al. performed an intraoperative and postoperative comparison of wounds - incisions applied to skin in neck dissection using an ultrasound scalpel (15 patients) and a monopolar electrocautery (15 patients), which were standard equipment for soft tissue surgery [5]. It is shown that ultrasonic scalpel led to a decrease in bleeding in the incision and postoperative pain, while the operation time and complications in both groups were the same. In another study, a 4-year, randomized trial was performed to evaluate the effectiveness of an ultrasound scalpel after opening of the inguinal and axillary lymph nodes with a therapeutic purpose, in which results were compared after using an ultrasound scalpel with a control group (ligation and a monopolar electrocoagulator). The results showed that the use of ultrasonic scalpels did not lead to a significant reduction in intraoperative and early postoperative complications, did not shorten an operation time, and this could increase the rate of lymphedema growth [6]. Chen XL et al. performed a systematic review and metaanalysis of studies on the effectiveness of the use of an ultrasound scalpel with traditional methods (monopolar electrocautery and ligation) in open gastrectomy for patients with carcinoma of the stomach ( 7 randomized and 12 comparative studies, a total of 1,930 patients). The authors showed that, in comparison to traditional surgery, the use of an ultrasonic scalpe led to a reduction in the operation time in the average from 185 min to $151 \mathrm{~min}$, decrease in intraoperative blood loss from 218 $\mathrm{mL}$ to $112 \mathrm{~mL}$, and decrease in cumulative risk of postoperative complications from $12.9 \%$ to $8.9 \%$ [7].

Laser excision is a highly sensitive, painless, precise method of exposure, allowing to remove tissue at a given depth. Cut depth is 2-3 $\mathrm{mm}$; thus, the separation of tissues is usually performed in several ways, dissecting them layer by layer. The laser scalpel works using the energy of the laser beam on the biological tissue, which sharply raises the temperature (up to $400^{\circ} \mathrm{C}$ ) in its limited area of about $0.001 \mathrm{~mm}$ in width. Such a pinpoint laser effect is fundamentally different from the mechanical action of a metal scalpel. As a result, the irradiated area instantly burns out, and partially evaporates. The effect of laser radiation is the coagulation of proteins, conversion of tissue fluid to the gaseous state, local destruction and burnout of the irradiated site. At the present time, dozens of types of lasers designed for performing various surgical operations have been developed: $\mathrm{CO}_{2}$ lasers, diode lasers, free electron lasers and others.

$\mathrm{Qu}$ W. et al. in a 30-day comparative study on the healing of the mucous membrane of the palate in rats showed that incision made with a laser had a less inflammatory response and minimal tissue damage compared with the traditional scalpel [8].

Histological and immunohistochemical analysis of cut wounds inflicted to guinea pigs by scalpel, CO2-laser and YSGG laser showed that the $\mathrm{CO} 2$ laser had better hemostasis ability, but its use led to more tissue damage than the scalpel and YSGG laser [9] The histological picture and the level of TNF-alpha expression in all wounds were comparable. The authors concluded that the YSGG laser had many advantages for oral surgery due to low inflammatory response and minimal tissue damage.

Suragimath $\mathrm{G}$ et al. conducted a randomized clinical comparative study to assess the effectiveness of the procedure for depigmentation of the gums by using the conventional scalpel and diode laser $(980 \mathrm{~nm})$ and showed that depigmentation of the gums was equally effective in both the scalpel and laser methods. However, the areas treated with a laser caused less pain in patients [10]. Alhabashneh R. et al. conducted a 6-month trial and showed equal efficacy in the treatment of hyperpigmentation of the gums with a laser and a scalpel, postoperative pain perception and the time required for treatment were also the same, with laser therapy requiring more advanced technology and higher financial costs [11]. The data obtained explain that the scalpel is still the gold standard for the treatment of gingival dysgenesis.

Concerning the treatment of fibrous hyperplasia of the oral cavity, laser cutting devices have not shown much success. In one randomized clinical study, surgical and postoperative assessments were analyzed for 3 weeks after surgical treatment of fibrous hyperplasia of the oral cavity with a diode laser and a metal scalpel. There are significant differences in the duration of the operation and the use of analgesic drugs. The use of a diode laser proved to be more effective and less invasive, but the healing time was faster with the scalpel [12].

Thus, the achievements of modern applied physics and the resulting clinical outcomes could not convince plastic surgeons in undeniable advantages in ultrasonic and laser cutting devices.

Electrosurgery is often used in dermatological practice to ensure superficial or deep coagulation and skin incision. The skin has poor conductive properties for the electrical energy flow, thus, this energy is stored and converted into heat. The essence of the method lies in the destruction of biological tissues by converting an alternating electric current frequency from $200 \mathrm{kHz}$ to a megahertz (usually $200-800 \mathrm{kHz}$ ) into thermal energy. As a result, two methods of electrosurgery are possible: 1 - electrocoagulation (burning) and protein coagulation (diathermocoagulation); and 2 electrotomy (dissection and excision of tissues). The effect of electrosurgery depends on the shape of the wave. For tissue coagulation, the pulsed mode of the electrosurgical instrument is used, for dissection - continuous. The incisions can be performed in two ways: electroacutomy (the rapid conduct of a thin electrode forms a very thin layer of coagulated tissues that do not prevent the healing of the wound by primary tension) and electrocoagulotomy (performed by slower conduction of a thicker echinoderm, such a wound is better coagulated, but heals by a slower secondary tension because of deeper coagulation lesions of the wound edges).

Advantages of electrosurgery can be expressed in reducing blood loss and reducing the size of the injured surface. Unfortunately, the issues of the clinical efficacy of electrosurgery still remain controversial.

In randomized blinded control trial Scott J.E. et al. determined the effect of monopolar electrosurgery in the 10,20 or $30 \mathrm{~W}$ mode for the duration of the operation, hemostasis and healing of cutaneous wounds compared to the scalpel incisions. The results of the study showed that the use of monopolar electrosurgery in different 
cutting regimens improved hemostasis and shortened the operation time for skin cutting in animals ( 15 dogs), but delayed healing and increased complications during the first 7 days compared to the scalpel incisions [13].

Ismail A. et al. conducted a systematic review and metaanalysis of work comparing electrocautery cutters and surgical incisions with a scalpel [14]. The analysis included 48 studies (including 36 randomized) with the participation of 6,422 patients. It was confirmed that electrocoagulation, in contrast to the incision with an ordinary surgical scalpel, was accompanied by a decrease in blood loss, a decrease in the incision time, and reduction in pain In this case, scars after the cut with an electron knife and a scalpel were visually the same.

Cochrane survey of randomized controlled trials comparing electrosurgery with a traditional metal scalpel to create abdominal incisions during open abdominal surgery (16 studies, 2,769 participants) showed that there were no clear differences in wound infections between electrosurgery and a scalpel $(7.7 \%$ in case of electrosurgery, $7.4 \%$ in case of a scalpel) [15]. None of the included studies reported time to heal wounds. In addition, there were no clinically significant differences in the time of the incision and the level of blood loss. Due to the heterogeneity of the groups, it was not possible to determine the effect of the two interventions on pain and on the characteristic appearance of scars. The authors came to the conclusion about the need for additional studies that would be correctly structured from the position of evidence-based medicine.

Thus, it is recognized that the important advantages of electrosurgery as compared to the scalpel are hemostasis (stopping the bleeding of small vessels), blockade of the lymphatic tract, and reduction of pain in the wound (due to coagulation of the intersected nerves). However, the final results of electrosurgery did not affect the size and depth of the postoperative scar. The main factors influencing the resulting effect of electrosurgery are the contact time of electrodes with tissue (duration of exposure), size and shape of the contact electrodes, and the waveform. In addition, the frequency of the current is of great importance.

The use of waves at a high frequency $(2-6 \mathrm{MHz})$ was called radiofrequency electrosurgery (radio knife). Since the radio knife works by the flow of alternating electric current at the much higher frequency, heating and tissue damage in the cut zone is minimal. The excision of tissues is caused by the creation of a point high-temperature zone at the point where the electrode contacts with tissue. That is, in fact the cut is carried out by thermal destruction, and the electric current acts only as a means for heating and does not affect tissue damage. The radio knife accelerates the movement of water molecules, i.e. mainly affects intracellular and intercellular fluids that boil and evaporate, providing coagulation and sterilization of surrounding tissues. Tissues with low water content are virtually not damaged. Consequently, the radiofrequency scalpel is a minimally invasive tool that allows to cut and coagulate tissues atraumatically, delivering heat energy directly to the target area. This allows to place high hopes on radiofrequency knives in surgery in general, especially in aesthetic surgery.

Thus, J. Niamtu published a 30-year experience in the use of radio wave surgery $(4 \mathrm{MHz})$ for aesthetic removal of head and neck nevi, as well as associated benign lesions of the face [16]. The surgeon has shown that the technique of radiofrequency ablation is safe. It removes nevi with minimal scars painlessly. The same doctor conducted a comparative analysis of the aesthetic results in the blepharoplasty of the upper eyelid in 30 women performed in one eyelid by radiofrequency surgery (Surgitron, Ellman International, $4 \mathrm{MHz}, 12 \mathrm{~W}$ ), and in another eyelid - by an ultrapulse $\mathrm{CO}_{2}$ laser (Encore, Lumenis Inc.; continuous wave $8 \mathrm{~W}$ ). Without discussing the ethical problems of this type of the study, eyelids on both sides were equally aesthetically pleasing in $26 \%$ of cases, the side of radio wave surgery was better in $37 \%$, and the side with the use of $\mathrm{CO}_{2}$ laser - in $37 \%$ of cases [17]. Based on the results, the author concluded that radio wave surgery and the laser cut gave the same cut and coagulation. Both methods limit blood loss and reduce operative time.

Not all studies were so unanimous in relation to radio wave surgery. Hasar Z.B. et al. in an experiment on 42 Wistar rats made cuts on the mucous membrane of the roof of mouth with a scalpel, electrosurgical instrument or radiofrequency device. Electrosurgery and radiosurgery were superior to the scalpel for hemostasis, but the weight of the animals in these groups was less for 14 days after the operation, which was regarded as evidence of more severe pain. Tissue coagulation was greater in the radiofrequency group. However, immunohistochemical analysis showed that the inducible expression of nitric oxide synthase and heat shock protein 70 was the same in all three groups [18].

Despite the wide use of lasers, radio wave and power tools in aesthetic medicine, there are contradictory data in the literature, in particular, the influence of lateral thermal damages associated with these instruments on wound healing. Histological comparison of dog skin biopsies $(\mathrm{n}=4)$ collected using monopolar electrosurgery, $\mathrm{CO}_{2}$ laser, radio wave surgery (cut edges of biopsy specimens and adjacent peripheral skin were assessed using light microscopy to compare dermis penetration by carbonizing tissues) showed that radio waves led to less lateral thermal damage to the skin than monopolar electrosurgery and $\mathrm{CO}_{2}$ laser [19]. In another experimental study (5 pigs), it was found that with the cuts performed with a $\mathrm{CO}_{2}$ laser there was the maximum proliferation of epithelial cells and the most extensive zone of lateral thermal damage, with no significant differences between electrosurgery and radiosurgery groups. The inflammatory response and the detection of $\mathrm{Ki}-67$ as a marker of proliferation of epithelial cells were the greatest in the laser group, but they did not significantly differ from all other groups, including the scalpel [20]. The absence of differences in these works might be due to the insufficient number of animals.

\section{Conclusion}

The study of the published works over the past 10 years shows that the developers do not abandon attempts to offer new devices based on clinical principles of ultrasound laser, electricity of different frequency and wavelength to clinical surgery. The above methods are characterized by high coagulation ability, the implementation of thin incisions with coagulation of the wound edges, and safety of use in relation to early postoperative complications. However, the beauty of these devices has not yet fascinated practical surgeons and still most of them use mechanical scalpels in routine practice. An exception may be plastic surgeons, as well as dermatologists. It is very likely that this is a result of merely surgical rigidity. However, it seems to us that most of the mistrust is caused by incorrectly structured studies. Amazing is the fact that the number of works devoted to the use of special high-tech cutting devices in humans exceeds by far the number of works carried out on the laboratory animals. The tasks achieved by different types of research studies can be performed only if all the principles of evidence-based medicine are observed. Unfortunately, genetically pure lines on which it would be possible to conduct really comparative studies are available only in animals. An attempt to answer questions about which of the devices is better when used on people without evaluating hundreds of indicators that determine the patient's overall somatic state, skin type, and pre-, intra- and postoperative conditioning (ointments, medicines, patches, physiotherapy procedures) will always be doomed to the emergence of even more questions.

However, the prospects of devices using different physical principles do not cause doubts, but they require large, wellconducted studies from the position of evidence-based medicine, including multifactor analysis. 


\section{References}

[1] American Society of Plastic Surgeons. 2014 Complete Plastic Surgery Statistics Report. https://www.plasticsurgery.org/news/plastic-surgerystatistics?sub=2014+Plastic+Surgery+Statistics\#section-title

[2] Milothridis P, Pavlidis L, Haidich A \& Panagopoulou E (2016), A systematic review of the factors predicting the interest in cosmetic plastic surgery. Indian Journal of Plastic Surgery 49(3), 397-402.

[3] Frederick DA, Lever J \& Peplau LA (2007), Interest in cosmetic surgery and body image: Views of men and women across the lifespan. Plastic and Reconstructive Surgery 120:1407-1415.

[4] Barnes L, Marshall CD, Leavitt T, Hu MS, Moore AL, Gonzalez JG, Longaker MT \& Gurtner GC (2018), Mechanical Forces in Cutaneous Wound Healing: Emerging Therapies to Minimize Scar Formation. Advances in Wound Care (New Rochelle) 7(2), 47-56.

[5] Schneider D, Goppold K, Kaemmerer PW, Schoen G, Woehlke M \& Bschorer R (2018). Use of ultrasonic scalpel and monopolar electrocautery for skin incisions in neck dissection: a prospective randomized trial. Journal of Oral and Maxillofacial Surgery 22(2), 169-175.

[6] Matthey-Gié ML, Gié O, DS, Demartines N, Matter M (2016), Prospective Randomized Study to Compare Lymphocele and Lymphorrhea Control Following Inguinal and Axillary Therapeutic Lymph Node Dissection with or Without the Use of an Ultrasonic Scalpel. Annals of Surgical Oncology 23(5), 1716-20.

[7] Chen XL, Chen XZ, Lu ZH, Wang L, Yang K, Hu JK, Zhang B, Chen ZX, Chen JP \& Zhou ZG (2014), Comparison of ultrasonic scalpel versus conventional techniques in open gastrectomy for gastric carcinoma patients: a systematic review and meta-analysis. PLoS One 9(7), e103330.

[8] Qu W, Shang J, Liu L, Xu D, Du P \& Liu Z (2018), Comparative study on the incision healing of the palatal mucosa by using Er:YAG laser or traditional scalpel in the SD rats. Lasers in Medical Science 33(5), 1019-1024.

[9] Ryu SW, Lee SH, Yoon HJ (2012), A comparative histological and immunohistochemical study of wound healing following incision with a scalpel, CO2 laser or Er,Cr:YSGG laser in the guinea pig oral mucosa. Acta Odontologica Scandinavica 70(6), 448-454.

[10] Suragimath G, Lohana MH \& Varma S (2016), A Split Mouth Randomized Clinical Comparative Study to Evaluate the Efficacy of Gingival Depigmentation Procedure Using Conventional Scalpel Technique or Diode Laser. Lasers in Medical Science 7(4):227-232.

[11] Alhabashneh R, Darawi O, Khader YS \& Ashour L (2018), Gingival depigmentation using Er:YAG laser and scalpel technique: A six-month prospective clinical study. Quintessence International 49(2), 113-122.

[12] Amaral MB, de Ávila JM, Abreu MH \& Mesquita RA (2015), Diode laser surgery versus scalpel surgery in the treatment of fibrous hyperplasia: a randomized clinical trial. International Journal of Oral and Maxillofacial Surgery 44(11), 1383-1389.

[13] Scott JE, Swanson EA, Cooley J, Wills RW \& Pearce EC (2017), Healing of canine skin incisions made with monopolar electrosurgery versus scalpel blade. Veterinary Surgery 46(4), 520529.

[14] Ismail A, Abushouk AI, Elmaraezy A, Menshawy A, Menshawy E, Ismail M, Samir E, Khaled A, Zakarya H, El-Tonoby A \& Ghanem E (2017), Cutting electrocautery versus scalpel for surgical incisions: a systematic review and meta-analysis. Journal of Surgical Research 220, 147-163.

[15] Charoenkwan K, Iheozor-Ejiofor Z, Rerkasem K \& Matovinovic E (2017), Scalpel versus electrosurgery for major abdominal incisions Cochrane database of systematic reviews 6, CD005987.

[16] Niamtu J (2014), Esthetic removal of head and neck nevi and lesions with 4.0-MHz radio-wave surgery: a 30-year experience. Journal of Oral and Maxillofacial Surgery 72(6), 1139-1150

[17] Niamtu J (2008), Radiowave surgery versus CO laser for upper blepharoplasty incision: which modality produces the most aesthetic incision? Dermatologic Surgery 34(7):912-921.

[18] Hasar ZB, Ozmeric N, Ozdemir B, Gökmenoğlu C, Baris E, Altan G \& Kahraman S (2016), Comparison of Radiofrequency and Electrocautery with Conventional Scalpel Incisions. Journal of Oral and Maxillofacial Surgery 74(11), 2136-2141.

[19] Silverman EB, Read RW, Boyle CR, Cooper R, Miller WW \& McLaughlin RM (2007), Histologic comparison of canine skin biopsies collected using monopolar electrosurgery, $\mathrm{CO} 2$ laser, radiowave radiosurgery, skin biopsy punch, and scalpel. Veterinary Surgery 36(1), 50-56.
[20] Schoinohoriti OK, Chrysomali E, Tzerbos F \& Iatrou I (2012), Comparison of lateral thermal injury and healing of porcine skin incisions performed by $\mathrm{CO} 2$-laser, monopolar electrosurgery and radiosurgery: a preliminary study based on histological and immunohistochemical results. International Journal of Dermatology 51(8), 979-986. 